\title{
HYDROPHOBIZATION OF TRACK MEMBRANE SURFACE BY MAGNETRON SPUTTER DEPOSITION OF ULTRA-HIGH MOLECULAR WEIGHT POLYETHYLENE
}

\author{
L. Kravets ${ }^{a, *}$, V. Altynov ${ }^{a}$, R. Gainutdinov ${ }^{b}$, N. Lizunov ${ }^{a}$, V. Satulu ${ }^{c}$, \\ B. $\operatorname{Mitu}^{c}$, G. Dinescu ${ }^{c}$ \\ a Joint Institute for Nuclear Research, Flerov laboratory of Nuclear Reactions, Joliot-Curie Str. 6, 141980 \\ Dubna, Russia \\ b Shubnikov Institute of Crystallography of FSRC "Crystallography and Photonics" of RAS, Leninskii Pr. 59, \\ 119333 Moscow, Russia \\ ${ }^{c}$ National Institute for Laser, Plasma and Radiation Physics, Atomistilor Str. 409, 077125 Magurele, Bucharest, \\ Romania \\ * kravets@jinr.ru
}

\begin{abstract}
Method for the formation of polymer coatings on the poly(ethylene terephthalate) tracketched membrane surface by magnetron sputter deposition of ultra-high molecular weight polyethylene in a vacuum is considered. The surface morphology and chemical structure of nanoscale coatings have been investigated. It is shown that the application of the ultra-high molecular weight polyethylene-like coatings leads to hydrophobization of the membrane surface, the degree of which depends on the coating thickness. Besides, the usage of this modification method leads to smoothing of structural inhomogeneity of the membrane surface, a decrease in pore diameter, and alteration of pore shape. The investigation of the chemical structure of deposited coatings by XPS method showed that they contain a significant concentration of oxygen-containing functional groups. The composite membranes of the developed sample can be used in the process of desalination of seawater by the method of membrane distillation.
\end{abstract}

Keywords: track-etched membrane, magnetron sputter deposition of polymers, ultra-high molecular weight polyethylene, hydrophobization, bilayer composite membranes.

\section{Introduction}

Currently, it is of great interest to develop methods for creating bilayer composite membranes (BCM), in which one of the layers has a highly porous hydrophilic base, and the second thin microporous layer has hydrophobic properties. In recent papers, it was shown that membranes of this type are also promising along with the common hydrophobic membranes made of polypropylene, polyvinylidene fluoride, and polytetrafluoroethylene in membrane distillation processes for desalination of seawater $[1,2]$. The usage of a thin hydrophobic layer combined with a thick hydrophilic substrate allows increasing the permeate flow through the membrane by decreasing the resistance against mass transfer. There are different methods of creating BCM; deposition of a polymer layer synthesized by plasma is one of these techniques $[3,4]$. The usage of plasma demonstrates important advantages: it allows controlling the thickness of the deposited polymer layer, providing high adhesion; a minor of conducting the process also characterizes it and the possibility to use a wide range of organic and organoelement compounds. The usage of this method for the polymerization of organic compounds containing functional groups allowed obtaining hydrophilic polymer layers on the membrane surface [5].
However, the attempt to use the method of plasma polymerization for the obtainment of the hydrophobic polymer layer on the membrane surface has not been successful. The studies showed that functional groups containing oxygen, in particular, carboxylic groups, are formed on the surface of the deposited layers at the usage of precursors without functional groups (for example, acetylene [6] or 1,1,1,2-tetrafluoroethane [7]). Such groups are formed when the samples are taken into the air; the presence of residual oxygen in the vacuum reaction chamber also causes their formation [8]. The insufficiently high values of water contact angles are related to the formation of the groups containing oxygen in the surface layer of the deposited polymers. In this regard, the search for new methods for the synthesis of bilayer composite membranes, in which one of the layers has a hydrophobic nature, is very relevant. A range of modern methods can be used for the deposition of thin hydrophobic layers onto the surface of porous membranes: chemical vapor deposition, sol-gel technique, photochemical grafting, etc. However, the formation of coatings from the active gaseous phase by the dispersion of polymers under, for example, laser radiation, $\mathrm{RF}$-discharge plasma, or a beam of accelerated electrons attracts the highest interest among them. These methods are high-tech; 


\begin{tabular}{|c|c|c|c|c|}
\hline \multirow{2}{*}{ Parameters } & \multirow{2}{*}{$\begin{array}{l}\text { Initial } \\
\text { PET TM }\end{array}$} & \multicolumn{3}{|c|}{ Thickness of deposited UHMWPE-like coating } \\
\hline & & $50 \mathrm{~nm}$ & $75 \mathrm{~nm}$ & $100 \mathrm{~nm}$ \\
\hline Relative increase in the mass, $\%$ & - & 7.4 & 10.0 & 11.7 \\
\hline 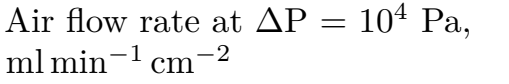 & 60 & 50 & 45 & 40 \\
\hline Effective pore diameter, nm & 65 & 62 & 60 & 57 \\
\hline Water contact angle, deg & 65 & 78 & 82 & 94 \\
\hline $\begin{array}{l}\text { Surface roughness } R_{\mathrm{ms}}, \mathrm{nm} \\
\left(1 \times 1 \mathrm{~m}^{2} \text { scan area }\right)\end{array}$ & 23.2 & 13.8 & 8.0 & 5.2 \\
\hline $\begin{array}{l}\text { Surface roughness } R_{\mathrm{ms}}, \mathrm{nm} \\
\text { (area without pores) }\end{array}$ & 5.4 & 4.0 & 2.8 & 3.5 \\
\hline
\end{tabular}

Table 1. Change of the PET TM characteristics after applying of UHMWPE-like coatings with different thicknes on its surface.

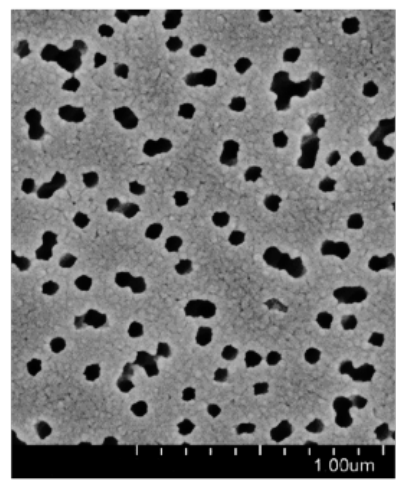

(a)

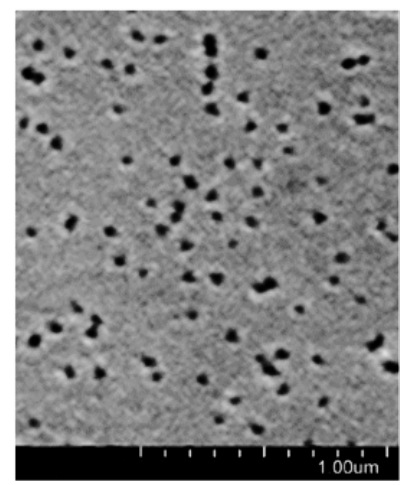

(b)

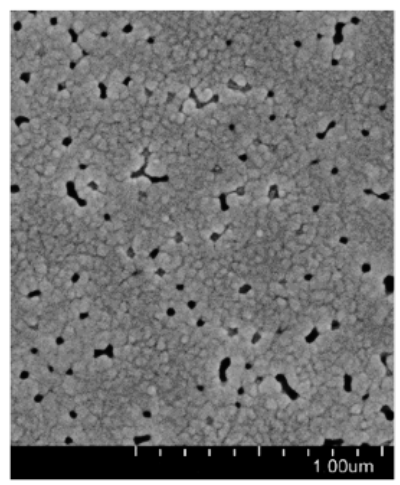

(c)

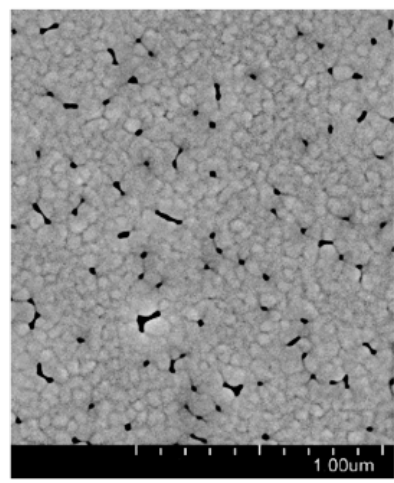

(d)

Figure 1. SEM images of the (a) initial PET TM surface and membranes with coating of UHMWPE-like with a thickness of (b) 50, (c) 75 and (d) $100 \mathrm{~nm}$ deposited by magnetron sputtering of original polymer.

they allow controlling the structure and composition of the deposited layers and obtaining superhydrophobic coatings on the surface of solids $[9,10]$.

In this work, the results on the synthesis and characterization of bilayer composite membranes containing a thin hydrophobic layer and thick hydrophilic substrate are reported. In order to obtain these membranes a thin coating from ultra-high molecular weight polyethylene (UHMWPE) was applied on the surface of a poly(ethylene terephthalate) track-etched membrane (PET TM) by magnetron sputter deposition method.

\section{Experimental}

In the present experiments, poly(ethylene terephthalate) track-etched membrane (PET TM) with an effective pore diameter of $65 \mathrm{~nm}$ and pore density of $3 \times 10^{9} \mathrm{~cm}^{-2}$ was used. To produce the membrane, the PET film with a thickness of $10.0 \mu \mathrm{m}$ (Lavsan, Russia) was irradiated by krypton positive ions, accelerated to energy of $3 \mathrm{MeV}$ per nucleon at the cyclotron of the Flerov Laboratory of Nuclear Reactions. Then the ion-irradiated film was additionally sensitized with UV irradiation with the maximum emission intensity at $310 \mathrm{~nm}$. The chemical etching process was per- formed in the aqueous solution of sodium hydroxide with concentration of $1.0 \mathrm{~mol} \mathrm{~L}^{-1}$ at $75^{\circ} \mathrm{C}$ according to the technique described in [11]. The membrane samples were cut in circular form with diameter of $10 \mathrm{~cm}$ prior the experiments.

The nanoscale layers of ultra-high molecular weight polyethylene-like (UHMWPE-like) coatings were deposited on the membrane surface from an active gas phase obtained by magnetron sputter deposition (MSD) of the original polymer in a vacuum. The deposition was conducted in a spherical stainless steel vacuum chamber evacuated by a turbomolecular pumping system down to a base pressure of $10^{-2} \mathrm{~Pa}$. The pressure in the chamber was monitored by a Pfeiffer gauge and the flow rates are controlled by electronic mass flow controller (Bronkhorst Instrum.). The chamber is equipped with a magnetron sputtering source (Kurt J. Lesker) and a second capacitively-coupled plasma source, as well with other ports for various plasma diagnostic systems. The magnetron source was mounted at $45^{\circ}$ and of $6 \mathrm{~cm}$ distance in respect to the substrate holder, which serves also as grounded electrode. The substrate holder is rotating during the deposition in order to insure thickness uniformity over large area. The UHMWPE target mounted in 


\begin{tabular}{|c|c|c|c|}
\hline \multirow{2}{*}{ Coating thickness } & \multicolumn{2}{|c|}{ Atomic content, at.\% } & \multirow{2}{*}{$\begin{array}{l}\text { Atomic ratio } \\
\text { O/C }\end{array}$} \\
\hline & $\mathrm{C}$ & $\mathrm{O}$ & \\
\hline $50 \mathrm{~nm}$ & 85.8 & 14.2 & $1.65 \times 10^{-1}$ \\
\hline $75 \mathrm{~nm}$ & 88.9 & 11.1 & $1.25 \times 10^{-1}$ \\
\hline $100 \mathrm{~nm}$ & 84.9 & 15.1 & $1.80 \times 10^{-1}$ \\
\hline
\end{tabular}

Table 2. XPS data on the elemental composition of UHMWPE-like coatings with different thicknesses deposited by magnetron sputtering of original polymer.

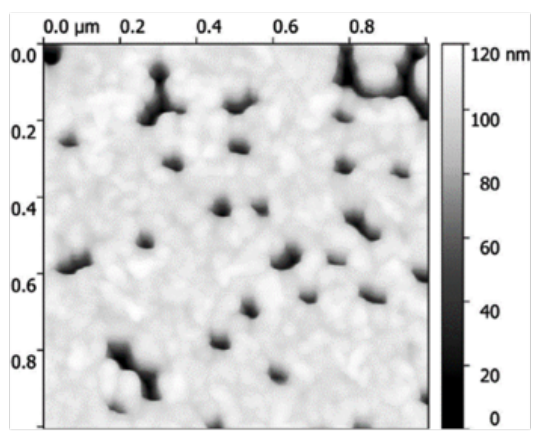

(a)

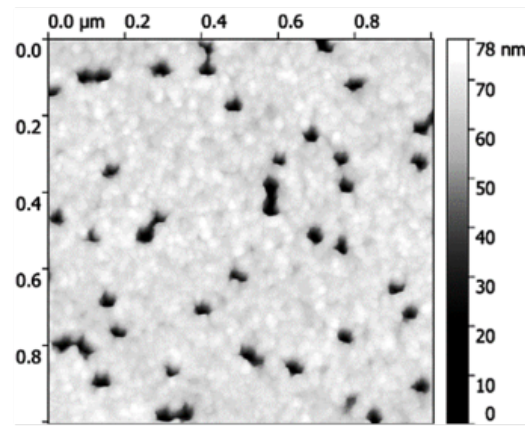

(b)

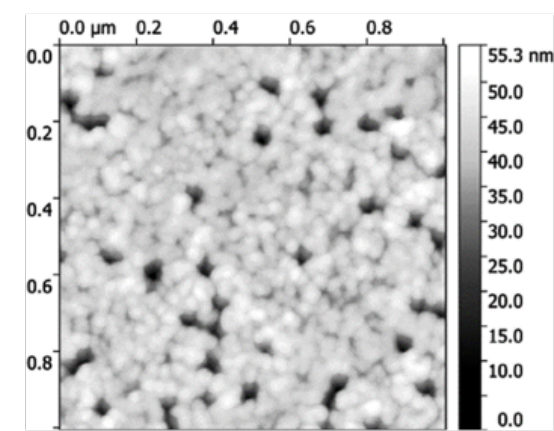

(c)

Figure 2. AFM images of the PET TM surface after applying of UHMWPE-like coating with a thickness of (a) 50, (b) 75 and (c) $100 \mathrm{~nm}$ deposited by magnetron sputtering method.

the magnetron is sputtered by igniting a discharge in argon flow (100 sccm) at RF power of $50 \mathrm{~W}$ and working pressure of $1.8 \times 10^{-1} \mathrm{~Pa}$. The deposition rate of the UHMWPE-like coating was $50 \mathrm{~nm} / \mathrm{hr}$. In order to insure various thicknesses of the UHMWPE-like layers, the deposition time was varied. The determination of UHMWPE-like layers thickness was controlled by contact profilometry method by using a Tenkor profilometer. The schematic of the setup and the MSD coating procedures are detailed in [12].

The characteristics of the initial membrane and membranes with the deposited polymer layers were determined by different procedures. The amount of the deposited polymer on the membrane surface was defined by the gravimetric method. The gas flow rate through the membranes was determined at an adjusted pressure drop. Gas consumption was measured by a float-type flow meter. From the obtained data, the effective pore diameter was calculated using the Knudsen equation [13]. The study of the microstructure of samples and the determination of the diameter of pores on the membrane surface was performed using a HITACHI SU-8020 high resolution scanning microscope (Japan). A thin layer of gold was sprayed in a vacuum onto the samples before the examination. The surface properties were characterized by values of the water contact angle measured with an Easy Drop DSA100 instrument (KRUSS, Germany) and the software Drop Shape Analysis V.1.90.0.14 using deionized water as a test liquid (error $\pm 1^{\circ}$ ).

Surface topography of the membranes was studied using a NTEGRA Prima (NT-MDT Spectrum
Instruments, Russia) atomic force microscope (AFM). Measurements were made in the tapping mode using HA_FM silicon probes (Tipsnano, Estonia). The scanning area was $1 \times 1 \mathrm{\mu m}^{2}$; the resolution was $512 \times 512$ dots per image. The scans of smaller area without pores were studied to exclude the influence of pores on the determination of surface roughness. The main determined parameter was the surface roughness a root-mean-square deviation of the surface profile $\left(R_{\mathrm{ms}}\right)$, calculated over all scan points.

The chemical composition of the composite membrane was investigated by using the X-ray Photoelectron Spectroscopy (XPS) method. XPS-spectra were obtained using a Thermo Scientific K-Alpha spectrometer (USA) equipped with a hemispherical analyzer. X-ray radiation of an aluminum anode $\left(\mathrm{Al} K_{\alpha}=\right.$ $1486.6 \mathrm{eV}$ ) was used for photoelectron excitation at the tube voltage of $12 \mathrm{kV}$ and emission current of $3 \mathrm{~mA}$. The position of peaks was calibrated using a reference $\mathrm{C} 1 \mathrm{~s}$ peak $(284.6 \mathrm{eV})$ [14]. The general spectra were registered in a transmission band of $100 \mathrm{eV}$ with the step of $0.5 \mathrm{eV}$; the regional spectra were registered in a transmission band of $20 \mathrm{eV}$ with the step of $0.05 \mathrm{eV}$. The spectra were registered and processed using Advantage software; the shape of peaks was approximated by the convolution of the Gaussian and Lorentzian functions.

\section{Results and discussion}

The results of measurements of the characteristics of the initial PET TM and membranes after applying coatings of various thicknesses by magnetron sput- 


\begin{tabular}{lllll}
\hline \hline \multirow{2}{*}{ Coating thickness } & \multicolumn{4}{c}{ Relative content of chemical bonds, \% } \\
\cline { 2 - 5 } & $\mathbf{C}-\mathbf{C}, \mathbf{C}-\mathbf{H}$ & $\mathbf{C}-\mathbf{O}(\mathbf{H})$ & $\mathbf{C}=\mathbf{O}$ & $\mathbf{O}=\mathbf{C}-\mathbf{O}$ \\
\hline $50 \mathrm{~nm}$ & 75.7 & 15.1 & 6.6 & 2.6 \\
$75 \mathrm{~nm}$ & 74.9 & 19.1 & 4.4 & 1.6 \\
$100 \mathrm{~nm}$ & 73.8 & 17.9 & 6.0 & 2.3 \\
\hline \hline
\end{tabular}

Table 3. Distribution of carbon atoms according to chemical state in the C1s spectra of UHMWPE-like coatings of different thicknesses.

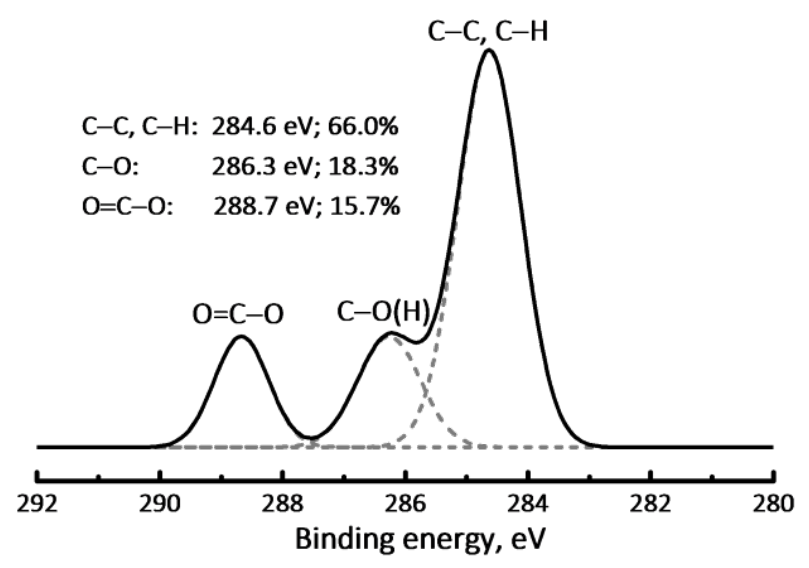

Figure 3. C1s spectrum of the initial PET TM.

tering of UHMWPE in vacuum on its surface are presented in Table 1. From the above data, it follows that the deposition of the UHMWPE-like coating leads to the increase in the weight of the membrane samples and a decrease in the effective pore diameter of the initial membrane. Reducing the effective pore diameter of the composite membranes ( $\mathrm{CMs})$ means that the pore diameter in the deposited polymer layer becomes smaller than the pore diameter in the initial membrane. Electron microscopic examination of the surface of modified membranes shows that for a membrane with a deposited UHMWPE-like layer of $50 \mathrm{~nm}$ thickness (Figure 1b) there is a slight decrease in the pore diameter on the surface. As the UHMWPE-like layer thickness increases, the pore diameter on the membrane surface decreases more significantly (Figures $1 \mathrm{c}$ and 1d). From electron microscopy data, it also follows that the pore diameter on the backside of the CMs remains unchanged. This indicates that the polymer deposition takes place only on the modified surface of the membrane. The deposition of the UHMWPE-like layer on the surface of PET TM by MSD method thus leads to the formation of composite membranes having an asymmetric shape of pore: the pore diameter on the untreated side of the membrane does not change, while the diameter of pores on the modified side decreases. This result correlates with the data we previously obtained during the study of PET TM modification by plasma polymerization [5-7].

AFM study of the topography of the track-etched membrane surface with a coating deposited from the active gas phase obtained by magnetron sputtering of UHMWPE (Figure 2), shows that the deposition of the polymer coating on the membrane surface leads to a change in its roughness. Thus, for the initial membrane, the $R_{\mathrm{ms}}$ value calculated for all scan points with an area of $1 \times 1 \mu^{2}$ is equal to $23.2 \mathrm{~nm}$ (Table 1). This relatively high value is determined by both the presence of pores on the membrane surface and the preparation technique of the track-etched membrane which includes chemical etching [11]. The presence of pores exhibits a more significant influence on the value of roughness. This fact is proven by the determination of such parameters as the average maximum peak height $\left(R_{\mathrm{pm}}\right)$ and the average valley depth $\left(R_{\mathrm{vm}}\right)$ of the surface profile. The estimation of these parameters for the initial membrane shows that the value of $R_{\mathrm{vm}}$ is $129.5 \mathrm{~nm}$, while the value of $R_{\mathrm{pm}}$ is $25.8 \mathrm{~nm}$, i.e., significantly lower. To avoid the influence of pores when determining surface roughness, scans with a smaller area that do not contain pores were processed. For the initial track-etched membrane, the $R_{\mathrm{ms}}$ value in this case is $5.4 \mathrm{~nm}$; this value is an order of magnitude lower than the one obtained for the scan of the larger area. Deposition of UHMWPE-like coating on the surface of PET TM leads to smoothing of the surface irregularities. The $R_{\mathrm{ms}}$ value of the modified membrane samples, calculated for all scan points with an area of $1 \times 1 \mathrm{\mu m}^{2}$, is lower than for the initial membrane (Table 1). This result is explained by the formation of the polymer in the pore channels at a certain depth from the entrance and partial blockage of the pores on the surface of the modified membranes. A decrease in the $R_{\mathrm{ms}}$ value on the pore-free surface during deposition also indicates that the UHMWPE-like coating has a smoother surface than the original membrane surface.

The study of the wettability of composite membranes shows that a hydrophobization takes place at the deposition of the UHMWPE-like coating on the surface of track-etched membrane. The initial membrane is characterized by the water contact angle $(\Theta)$ of $65^{\circ}$, then for PET TM coated with a polymer layer $50 \mathrm{~nm}$ thick, the $\Theta$ value is $78^{\circ}$. For a membrane with a UHMWPE-like layer with a thickness of $75 \mathrm{~nm} \Theta=$ $82^{\circ}$. Increasing the thickness of the applied polymer layer to $100 \mathrm{~nm}$ increases the water contact angle to $94^{\circ}$. Note, the values of the contact angles for these 

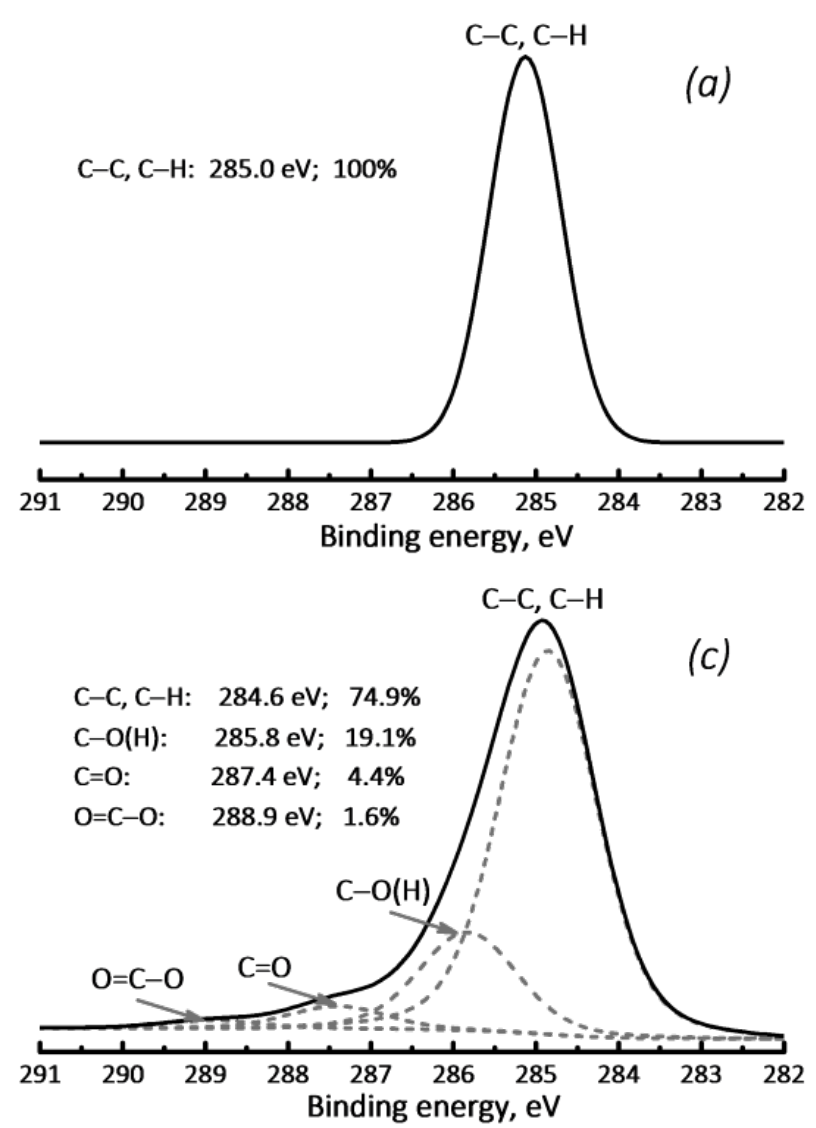
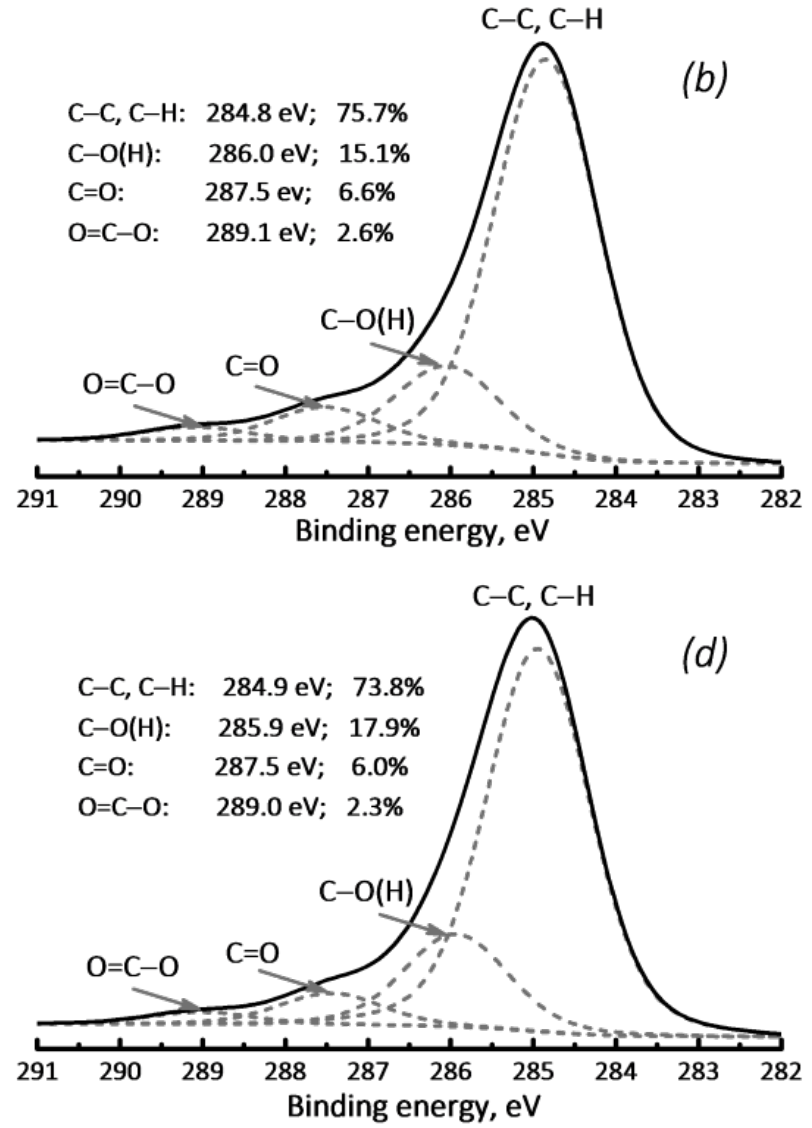

Figure 4. C1s XPS spectra of the (a) original UHMWPE and the UHMWPE-like coatings with a thickness of (b) 50, (c) 75 and (d) $100 \mathrm{~nm}$ deposited by magnetron sputtering of original polymer.

coatings are lower than for UHMWPE-like coatings obtained by the method of electron-beam deposition (EBD) [15]. Such changes are most likely due to differences in the chemical structure of polymer coatings. To obtain information about the changes in the chemical structure of the surface layer of membranes taking place during the deposition of coatings on the surface of the initial track-etched membrane, XPS spectra were registered.

Analysis of the surface layer spectrum of the initial PET TM (Figure 3) demonstrates the presence of lines attributed to carbon and oxygen atoms; their atomic concentrations are $74.5 \%$ and $25.5 \%$, respectively. The spectrum of carbon atom was studied in detail to determine the nature of the functional groups on the membrane surface. The decomposition of C1s spectral line of PET TM into components shows its complex character. The most intense spectral component characterized by the maximum of binding energy $(E)$ at $284.6 \mathrm{eV}$ and relative contribution of $66.0 \%$ corresponds to the carbon in the $\mathrm{C}-\mathrm{C}$ and $\mathrm{C}-\mathrm{H}$ bonds. The components characterized by the maxima of binding energy at 286.3 and $288.7 \mathrm{eV}$ are attributed to the carbon in the oxygen-containing $\mathrm{C}-\mathrm{O}(\mathrm{H})$ functional groups $(18.3 \%)$ and $\mathrm{O}=\mathrm{C}-\mathrm{O}(15.7 \%)$, respectively. The significant concentration of the oxygen-containing functional groups in the surface layer of the initial
PET TM determines the hydrophilicity of its surface.

The XPS study of the chemical structure of UHMWPE-like coatings deposited by the MSD method showed the presence of peaks attributed to carbon and oxygen atoms too (Table 2). A detailed analysis of $\mathrm{C} 1 \mathrm{~s}$ spectra indicates certain differences in the electron structure and chemical state of carbon in the original UHMWPE (Figure 4a) and deposited coatings (Figures 4b-d). A single intense peak characterized by the maximum at $E=285.0 \mathrm{eV}$ is present in the spectrum of the original UHMWPE; it corresponds to the chemical state of carbon in the $\mathrm{C}-\mathrm{C}$ and $\mathrm{C}-\mathrm{H}$ bonds. Four components are observed in the spectra of the UHMWPE-like coatings obtained by MSD method of this polymer. An intense one (maximum at $E=285.0 \mathrm{eV}$ ) corresponding to the chemical state of carbon in the $\mathrm{C}-\mathrm{C}$ and $\mathrm{C}-\mathrm{H}$ bonds. There are the components of lower intensity in the spectra: with a maximum binding energy of $286.0 \mathrm{eV}$, which corresponds to carbon in the $\mathrm{C}-\mathrm{O}(\mathrm{H})$ bonds, and with a maximum binding energy of $287.5 \mathrm{eV}$, corresponding to carbon in the $\mathrm{C}=\mathrm{O}$ functional groups. Another one is a component with a maximum binding energy of $289.1 \mathrm{eV}$, which corresponds to carbon in the $\mathrm{O}=\mathrm{C}-\mathrm{O}$ groups. These data indicate the emergence of oxygencontaining groups on the surface of the UHMWPE-like coatings. The ratio between the number of oxygen 
atoms and the number of carbon atoms in the deposited coatings is on average $1.6 \times 10^{-1}$, this value is two times less than on the surface of the initial PET TM, for which this ratio is $3.4 \times 10^{-1}$. This fact shows that the concentration of the ionogenic functional groups (particularly, oxygen-containing groups) on the surface of UHMWPE-like coatings is lower as compared to the initial membrane. The concentration of oxygen-containing groups corresponding to different chemical states of carbon in UHMWPE-like coatings of different thickness, as shown by experimental data, varies slightly (Table 3 ).

\section{Conclusions}

Thus, the applying the UHMWPE-like coatings to the surface of PET TM by the magnetron sputtering of original polymer leads to the formation of composite membranes consisting of two layers, one of which is the initial track-etched membrane, characterized by a medium level of hydrophilicity. The water contact angle of this layer is $65^{\circ}$. The other layer deposited by MSD method is more hydrophobic; the value of the water contact angle varies from $78^{\circ}$ to $94^{\circ}$ depending on the layer thickness. This layer contains a significant concentration of oxygen-containing functional groups. The insufficiently high values of water contact angles are related just to the formation of the oxygen-containing groups in the surface layer of the deposited polymers. Comparison of the experimental data obtained with the results of the study of polymer coatings from UHMWPE applied by electron-beam deposition shows that the coatings obtained by the EBD method are more similar in chemical composition to the original polymer. For them, there are a higher concentration of $\mathrm{C}-\mathrm{C}$ and $\mathrm{C}-\mathrm{H}$ chemical bonds and significantly lower concentrations of oxygen-containing groups compared to polymers deposited by magnetron sputtering. The composite membranes of the developed sample can be used in the membrane distillation processes for the desalination of seawater.

\section{Acknowledgements}

This research has been supported by a bilateral contract (no. 4648-5-17/2021) between JINR (Dubna, Russia) and NILPRP (Bucharest, Romania).

\section{References}

[1] M. Qtaishat, M. Khayet, and T. Matsuura. Guidelines for preparation of higher flux hydrophobic/hydrophilic composite membranes for membrane distillation. $J$. Membr. Sci., 329(1):193-200, 2009. doi:10.1016/j.memsci.2008.12.041.

[2] M. Essalhi and M. Khayet. Surface segregation of fluorinated modifying macromolecule for hydrophobic/hydrophilic membrane preparation and application in air gap and direct contact membrane distillation. J. Membr. Sci., 417:163-173, 2012. doi:10.1016/j.memsci.2012.06.028.
[3] M. Bryjak and I. Gancarz. Membrane prepared via plasma modification. In Membranes for membrane reactors: preparation, optimization and selection, chapter 25. Chichester (UK): John Wiley and Sons., 2011.

[4] L. I. Kravets, A. B. Gilman, and G. Dinescu. Modification of polymer membrane properties by lowtemperature plasma. Rus. J. Gener. Chem., 85(5):12841301, 2015. doi:10.1134/S107036321505045X.

[5] L. Kravets, S. Dmitriev, N. Lizunov, V. Satulu, B. Mitu, and G. Dinescu. Properties of poly(ethylene terephthalate) track membranes with a polymer layer obtained by plasma polymerization of pyrrole vapors. Nucl. Instr. Meth. B., 268(5):485-492, 2010. doi:10.1016/j.nimb.2009.11.014.

[6] L. I. Kravets, S. N. Dmitriev, V. Satulu, B. Mitu, and G. Dinescu. Structure and electrochemical properties of track membranes with a polymer layer obtained by plasma polymerization of acetylene. J. Phys.: Confer. Ser., 516(1):012006, 2014. doi:10.1088/1742-6596/516/1/012006.

[7] L. I. Kravets, A. B. Gilman, V. Satulu, B. Mitu, and G. Dinescu. Formation of diode-like composite membranes by plasma polymerization. Inorg. Mater. Appl. Res., 9(2):162-174, 2018. doi : 10.1134/S207511331802017X.

[8] H. Yasuda. Plasma polymerization. Academic Press, Orlando, Florida., 1985.

[9] A. A. Rogachev, S. Tamulevicius, A. V. Rogachev, M. A. Yarmolenko, and I. Prosycevas. The structure and molecular orientation of polytetrafluoroethylene coatings deposited from active gas phase. Appl. Surf. Sci., 255(15):6851-6856, 2009. doi:10.1016/j.apsusc.2009.03.004.

[10] M. Drabik, O. Polonskaya, O. Kylian, J. Cechvala, A. Artemenko, I. Gordeev, A. Choukourov, D. Slavinska, I. Matolinova, and H. Biederman. Syper-hydrophobic coatings prepared by RF magnetron sputtering of PTFE. Plasma Process Polym., 7(7):544-551, 2010. doi:10.1002/ppap. 200900164.

[11] P. Y. Apel and S. N. Dmitriev. Micro- and nanoporous materials produced using accelerated heavy ion beams. Adv. Natur. Sci.: Nanosci. Nanotechnol., 2(1):013002, 2011. doi:10.1088/2043-6262/2/1/013002.

[12] V. Satulu, B. Mitu, A. M. Pandele, S. I. Voicu, L. I. Kravets, and G. Dinescu. Composite polyethylene terephthalate track membranes with thin teflon-like layers: preparation and surface properties. Appl. Surf. Sci., 476:452-459, 2019. doi:10.1016/j.apsusc. 2019.01.109.

[13] M. Mulder. Basic principles of membrane technology. Dordrecht: Kluwer Acad. Publ., 1996.

[14] D. Briggs and J. T. Grant. Surface analysis by auger and $X$-ray photoelectron spectroscopy. Chichester: IM Publ., 2003.

[15] L. I. Kravets, M. A. Yarmolenko, A. A. Rogachev, R. V. Gainutdinov, V. A. Altynov, and N. E. Lizunov. Deposition of double-layer coatings for preparing composite membranes with superhydrophobic properties. High Temp. Mater. Proc., 23(1):77-96, 2019. doi:10.1615/hightempmatproc. 2019030269. 